\title{
Histone H3 Amino-Terminal Tail Phosphorylation and Acetylation: Synergistic or Independent Transcriptional Regulatory Marks?
}

\author{
C.J. Fry, ${ }^{*}$ M.A. Shogren-KnAaK, ${ }^{*}$ And C.L. Peterson \\ Program in Molecular Medicine, University of Massachusetts Medical School, Worcester, Massachusetts 01605
}

Eukaryotic cells package their large genomes into higher-order nucleoprotein complexes termed chromatin, creating a barrier to many nuclear processes that require access to the DNA. The fundamental unit of chromatin is the nucleosome, which consists of an octamer of core histone proteins (two each of histone $\mathrm{H} 2 \mathrm{~A}, \mathrm{H} 2 \mathrm{~B}, \mathrm{H} 3$, and $\mathrm{H} 4)$. Each histone octamer contains an $(\mathrm{H} 3-\mathrm{H} 4)_{2}$ tetramer flanked by two H2A-H2B dimers that form a nucleosome core that binds to and wraps 1.65 turns (147 bp) of superhelical DNA (Luger et al. 1997). This wrapping of DNA around the nucleosome core partially restricts access to DNA-binding proteins. In addition, histone proteins also contain nonstructured amino- and carboxy-terminal tail domains that protrude from the nucleosome core and may interact with neighboring nucleosomes and nucleosomal DNA to further restrict access to DNA-binding proteins. Individual nucleosomes are connected by 10-60 bp of "linker" DNA to form contiguous nucleosomal arrays that are condensed into highly compacted 100-400-nmthick fibers in interphase chromosomes. Chromosomes are further compacted during mitosis, condensing the DNA up to 10,000-fold (Hayes and Hansen 2001; Woodcock and Dimitrov 2001). How then do cells regulate the accessibility of their genomic DNA?

In the last several decades, we have begun to understand how cells regulate chromatin structure to allow accessibility of the DNA. One mechanism is provided by the ATP-dependent chromatin remodeling enzymes, which have been shown to increase the mobility of nucleosomes and to increase the accessibility of nucleosomal DNA to DNA-binding factors (Becker and Horz 2002). A second mechanism involves the posttranslational modification of the histone proteins. The aminoterminal tails of histones $\mathrm{H} 2 \mathrm{~A}, \mathrm{H} 2 \mathrm{~B}, \mathrm{H} 3$, and $\mathrm{H} 4$, in addition to the carboxy-terminal tail of histone H2A, are subject to numerous posttranslational modifications including phosphorylation, acetylation, ubiquitination, methylation, sumoylation, and ribosylation (Fischle et al. $2003 \mathrm{~b}$ and references within). Recent studies have even identified modifications of residues within the nucleosome core that are involved in regulating chromatin structure (Ng et al. 2002; van Leeuwen et al. 2002; Zhang et al. 2003). Posttranslational histone modifications may directly regulate $\mathrm{DNA} /$ chromatin compaction by altering

*These authors contributed equally to this work.
histone-DNA contacts or disrupting nucleosome-nucleosome interactions that mediate chromatin folding (Hayes and Hansen 2001). Alternatively, these modifications may regulate the recognition/binding motifs for other chromatin enzymes or binding proteins, providing a regulatory scaffold for transcription and other cellular processes that access DNA.

Here we focus on one of the earliest and least understood modifications linked to transcriptional regulation, phosphorylation of the histone $\mathrm{H} 3$ amino-terminal tail, and discuss several mechanisms by which phosphorylation is thought to regulate transcription. Specifically, we will present experiments addressing a recent model proposing that histone $\mathrm{H} 3$ phosphorylation and acetylation are coupled and discuss several alternative models by which $\mathrm{H} 3$ phosphorylation may contribute to the regulation of chromatin structure and transcription.

\section{EARLY EVIDENCE FOR HISTONE PHOSPHORYLATION: ACTIVATION OF IMMEDIATE-EARLY GENES}

Studies on immediate-early gene expression in mammalian cells provided some of the earliest evidence that chromatin plays a major regulatory role in transcription. When stimulated with growth factors, cytokines, or specific pharmacological agents, quiescent cells elicit a rapid response, involving the activation of intracellular signaling cascades leading to altered gene expression and cell growth. The first phase of gene activation following stimulation with mitogens involves the expression of immediate-early genes c-fos and c-jun. Upon stimulation, the MAP kinase cascade targets the phosphorylation of downstream transcriptional regulatory proteins that activate transcription of these genes. Early studies by Allfrey and colleagues found that the chromatin structure of the c-fos and c-myc genes assumes a more open confirmation upon gene activation (Allegra et al. 1987; Chen and Allfrey 1987), suggesting that the modification of chromatin structure may be a key step in activation; however, it was not clear whether the observed chromatin changes were a cause or consequence of transcription.

Mahadevan and coworkers sought to identify downstream components of the MAP kinase signaling cascade by phosphate labeling mouse fibroblasts and purifying proteins that become phosphorylated after induction with epidermal growth factor (EGF) and 12-0-tetrade- 
canoylphorbol 13-acetate (TPA) (Mahadevan et al. 1991). Surprisingly, the authors identified histone H3 as a key downstream target of the MAP kinase cascade, suggesting that histone $\mathrm{H} 3$ phosphorylation contributes to the activation of immediate-early genes. Phosphorylation of histone $\mathrm{H} 3$ was resistant to the transcriptional inhibitor $\alpha$-amanitin, suggesting that phosphorylation of $\mathrm{H} 3$ was not a consequence of transcription, but rather a regulatory signal delivered to chromatin upon stimulation with mitogens. This was later confirmed by studies showing that pretreatment of cells with kinase inhibitors or deletion of the histone $\mathrm{H} 3$ kinase reduced activation of c-fos and cjun upon mitogen stimulation (Soloaga et al. 2003). These studies provided the first evidence linking histone H3 phosphorylation to transcriptional activation.

Histone $\mathrm{H} 3$, in addition to histone $\mathrm{H} 1$, had long been known to be globally phosphorylated during mitosis when chromosomes are condensed (for a recent review, see Prigent and Dimitrov 2003). Unlike mitosis, histone H3 phosphorylation during immediate-early gene activation occurs on only a small fraction of nucleosomes and is targeted to active genes. Studies using fibroblasts established from Coffin-Lowry Syndrome (CLS) patients suggested that Rsk-2, a member of the pp90 ${ }^{\text {rk }}$ family of kinases implicated in cell growth control, was the downstream effector kinase responsible for phosphorylating histone $\mathrm{H} 3$ during activation of immediate-early genes (Sassone-Corsi et al. 1999). However, more recent studies strongly implicate the mitogen-stimulated kinases Msk1 and Msk2 (Wiggin et al. 2002; Soloaga et al. 2003). How does histone $\mathrm{H} 3$ phosphorylation, a mark associated with condensed chromosomes, facilitate transcriptional activation? The answers came much later with the advent of histone modification-specific antibodies and chromatin immunoprecipitation techniques.

\section{HISTONE H3 PHOSPHORYLATION AND ACETYLATION: EVIDENCE FOR COUPLED VERSUS INDEPENDENT MARKS}

In addition to phosphorylation, early studies showed that histone $\mathrm{H} 3$ was also acetylated at immediate-early genes during transcriptional activation (Allegra et al. 1987; Chen and Allfrey 1987). This acetylation is likely to be mediated by histone acetyltransferase (HAT) proteins such as $\mathrm{CBP} / \mathrm{p} 300$ or $\mathrm{P} / \mathrm{CAF}$, which have been shown to interact with numerous transcriptional activator proteins. Recent chromatin immunoprecipitation (ChIP) studies identified serine 10 as the phosphorylation site and lysines 9 and 14 as acetylation sites on the histone H3 amino-terminal tail that accompany activation of the cfos and c-jun genes (Cheung et al. 2000; Thomson et al. 2001). More importantly, these studies showed that phosphorylation and acetylation occur on the same histone H3 tails, suggesting that the two modifications might be linked.

There has been much debate over the last several years whether H3-S10 phosphorylation and K9/K14 acetylation are coupled or whether they have independent functions during transcriptional activation. One model sug- gests a synergistic mechanism for the two modifications, where prior S10 phosphorylation enhances subsequent K9/K14 acetylation (Cheung et al. 2000; Lo et al. 2000). This model is based in part on studies of the immediateearly genes. Cheung and coworkers found that after stimulation of $\mathrm{C} 3 \mathrm{H}$ cells with EGF, bulk histone H3 S10 phosphorylation precedes K9/K14 acetylation, suggesting a temporal pattern of these two modifications (Cheung et al. 2000). Furthermore, the authors suggest that pretreatment of cells with an inhibitor of the MAP kinase signaling pathway (PD98059) reduces both phosphorylation and acetylation of histone $\mathrm{H} 3$ at the c-fos promoter, suggesting that the two modifications may be linked. Strong support for the synergistic model comes from in vitro studies showing that H3-S10 phosphorylation stimulates the acetylation of a small peptide corresponding to the $\mathrm{H} 3$ amino-terminal tail by the catalytic HAT domain of yeast Gcn5p (Cheung et al. 2000; Lo et al. 2000). A very modest stimulation was also reported for the catalytic domains of PCAF and p300, and purified yeast NuA3 and SAGA HAT complexes (Lo et al. 2000).

Although H3-S10 phosphorylation was found to stimulate the activity of HAT proteins in vitro, it is still not clear whether histone $\mathrm{H} 3$ phosphorylation and acetylation are coupled during the activation of immediate-early genes. In an extensive study of c-jun activation, Thomson and colleagues show that prior treatment of cells with MAP kinase inhibitors SB 203580 (specific for MAP kinase 38) or H89 (specific for Msk1), or deletion of MSK1 and MSK2, severely reduces H3-S10 phosphorylation levels but has no effect on acetylation of histone $\mathrm{H} 3$ at the c-jun promoter following anisomycin stimulation (Thomson et al. 2001; Soloaga et al. 2003). Furthermore, ChIP experiments using modification-specific antibodies did not find phosphorylation preceding acetylation of histone $\mathrm{H} 3$ at the c-jun promoter during activation, rather H3-S10 phosphorylation and K9/K14 acetylation occurred simultaneously. Taken together, these later studies strongly suggest that H3-S10 phosphorylation and K9/K14 acetylation are marks deposited on histone H3 independently during activation of immediate-early genes.

Although the function of H3-S10 phosphorylation may be to enhance $\mathrm{K} 9 / \mathrm{K} 14$ acetylation in some instances, other studies clearly show that phosphorylation has additional acetylation-independent functions in transcriptional activation. Nowak and Corces (2000) show that during the heat shock response in Drosophila, H3-S10 phosphorylation, but not $\mathrm{K} 9 / \mathrm{K} 14$ acetylation, increases at the heat shock genes during activation. Furthermore, non-heat shock regions of polytene chromosomes show a decrease in H3-S10 phosphorylation upon transcriptional repression during heat shock. Taken together, these results suggest that in Drosophila, histone H3 phosphorylation plays a global role in transcriptional activation that is independent of acetylation.

Further support for a synergistic phosphorylation/ acetylation model comes primarily from studies in yeast. Mutation of histone $\mathrm{H} 3$ serine 10 to alanine reduces transcription levels of a few genes in yeast, including $\mathrm{HO}$, HIS3, and INO1 (Lo et al. 2000; Lo et al. 2001); however, 
we have found no changes in cell-cycle-dependent HO expression in an S10A mutant strain (C.J. Fry et al., unpubl.). During their studies of the INO1 gene, Lo and coworkers (2001) found that Snflp, a protein kinase previously implicated in the regulation of INO1 gene expression, was able to phosphorylate histone $\mathrm{H} 3$ on S10 in vitro. Deletion of the Snf1 kinase, or the Gen5p HAT, or mutation of histone $\mathrm{H} 3 \mathrm{~S} 10$ or $\mathrm{K} 14$ reduces the activation of INO1 when yeast cells are shifted from high- to lowinositol medium. Furthermore, deletion of Snflp or mutation of histone $\mathrm{H} 3 \mathrm{~S} 10 \mathrm{~A}$ greatly reduces the acetylation of K9/K14 by Gcn5p during activation. Taken together, these results invoke the synergistic phosphorylation/ acetylation model for the yeast INO1 gene. However, it is unclear whether histone $\mathrm{H} 3 \mathrm{~S} 10$ phosphorylation plays a role in the activation of any other Gcn5p-regulated genes.

\section{MECHANISM OF COUPLING ACETYLATION TO PHOSPHORYLATION: BINDING OF PHOSPHORYLATED TAILS}

Does histone phosphorylation directly enhance histone acetylation? If so, what is the mechanism for the coupling of these two events? At least three different models exist to explain transcriptional activation by H3-S10 phosphorylation, some of which are independent of acetylation (Fig. 1). In the first model (Fig. 1A), histone tail phosphorylation creates a binding site for a HAT complex, thereby facilitating histone acetylation. In the second model (Fig. 1B), H3 serine 10 phosphorylation disrupts higher-order chromatin structure to facilitate the binding of transcriptional regulators. In the third model (Fig. 1C), histone tail phosphorylation disrupts binding of inhibitor/repressor proteins, allowing the binding of positive regulators of transcription.

Initial studies focused on the ability of H3-S10 phosphorylation to directly recruit acetyltransferase enzymes. Many chromatin-associated proteins bind histone tails at acetyl-lysine and methyl-lysine residues using structurally conserved bromo- and chromodomains, respectively (Dhalluin et al. 1999; Nielsen et al. 2002). Furthermore, a number of phosphoserine- and phosphothreonine-binding domains exist, such as 14-3-3 proteins, WW domains, and forkhead-associated domains (Yaffe and Elia 2001). While these domains have not been shown to bind histone tails, it was expected that a similar phosphoserine, histone tail-binding domain would be found. Based on the cocrystal structure of Tetrahymena Gen 5 bound to an unphosphorylated histone $\mathrm{H} 3$ peptide (Rojas et al. 1999), it was proposed that arginine 164 of Gcn5 might interact electrostatically with the anionic oxygens of a phosphoserine 10 moiety, thereby increasing the affinity of Gcn5 for phosphorylated histone H3 tails (Cheung et al. 2000; Lo et al. 2000). In support of this hypothesis, kinetic analysis using the catalytic domain of yeast Gen5 and phosphorylated and unphosphorylated histone $\mathrm{H} 3$ tail peptides show that phosphorylation does enhance peptide acetylation by a factor of 6-10 (Cheung et al. 2000; Lo et al. 2000). This enhancement is due to an increase in the binding affinity, not turnover ef- ficiency, of Gen5 toward phosphorylated peptides. Furthermore, mutagenesis of the putative phosphate-binding residue, arginine 164, eliminated this phosphate-mediated stimulation of acetylation, suggesting that arginine 164 does contact the phosphoserine 10 residue. Genetic studies in yeast show that mutation of arginine 164 results in slow growth under some conditions, and a reduction in levels of transcription of some genes, including HIS3 and $\mathrm{HO}$, lending in vivo support for a coupled phosphorylation and acetylation mechanism (Lo et al. 2000).

In cells, histones exist within chromatin, and this context has the potential to modulate the interactions with histone tails. For example, histone tails mediate interaction between nucleosomes in folded nucleosomal arrays, and these interactions change the ability of histone tails to be acetylated (Hayes and Hansen 2001). Furthermore, in a cellular context, histone acetyltransferases do not function in isolation, but are instead part of larger protein complexes that influence histone acetyltransferase activity. It is known that Gcn5-containing complexes bind more tightly to nucleosomal substrates and acetylate a larger range of lysine residues on nuclesomal histone tails

\section{A.}

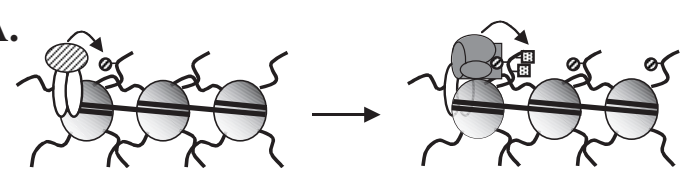

B.

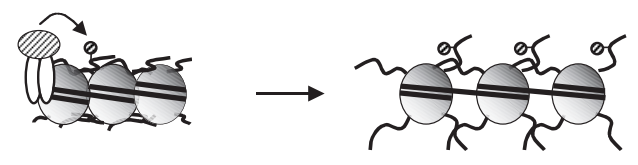

C.
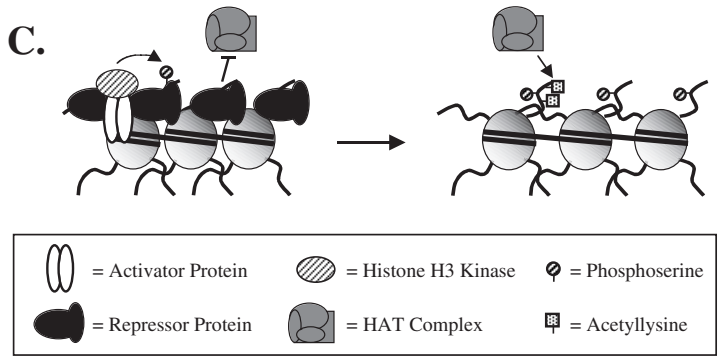

Figure 1. Possible mechanisms by which histone $\mathrm{H} 3$ phosphorylation may regulate chromatin structure and transcription. (A) Phosphorylation of $\mathrm{H} 3$ serine 10 by an activator-recruited histone H3 kinase such as MSK1 (left) may directly increase the binding affinity of the histone $\mathrm{H} 3$ amino-terminal tail for histone acetyltransferase proteins such as Gcn5, and enhance the acetylation of lysines 9 and 14 (right), leading to transcriptional activation. (B) Phosphorylation of histone H3 serine 10 (left) may disrupt histone-DNA or histone-histone interactions required for chromatin folding, leading to chromatin decondensation (right) and increased accessibility of the DNA to the transcriptional machinery. (C) H3 Serine 10 phosphorylation may remove a transcriptional repressor protein such as HP1 or INHAT from chromatin (left) by modifying a recognition motif on the histone $\mathrm{H} 3$ amino-terminal tail. Removal of the repressor protein may increase the accessibility of histone tails for further modification by histone acetyltransferase proteins (right), leading to transcriptional activation. 

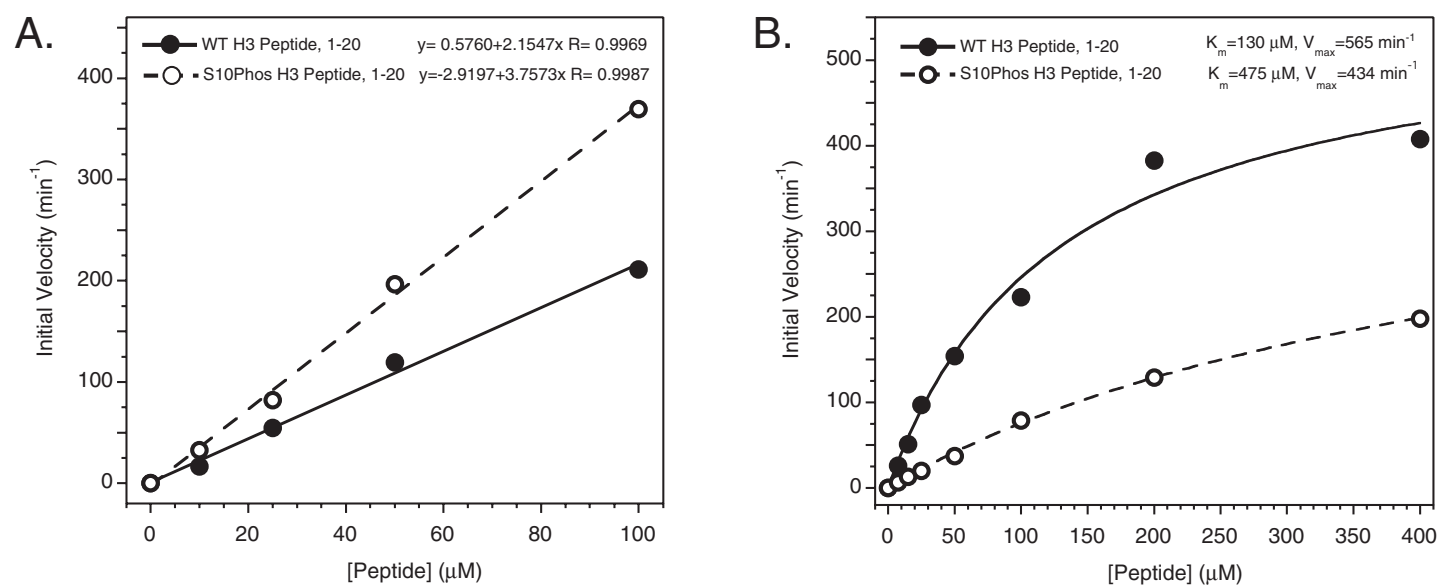

Figure 2. Serine 10 phosphorylation inhibits acetylation of a histone $\mathrm{H} 3$ peptide by the Gcn5p-containing SAGA complex. A comparison of the HAT activities of yeast $\operatorname{Gen} 5 \mathrm{p}(A)$ and SAGA complex $(B)$ on a serine 10 phosphorylated (open circles) or unphosphorylated (closed circles) histone $\mathrm{H3}$ amino-terminal peptide (amino acids 1-20). Histone acetyltransferase assays were performed with histone H3 peptide (0-400 $\mu \mathrm{M})$ and recombinant Gen5p (20-100 nM) or purified yeast SAGA complex (10-20 nM), as described previously (Shogren-Knaak et al. 2003). For each concentration of histone H3 peptide, the initial velocities were calculated by plotting the average incorporation of tritiated acetate as a function of time. Each assay was repeated at least twice and the average initial velocities are plotted as a function of peptide concentration. Recombinant yeast Gen5p (amino acids 99-262) and purified yeast SAGA complex were prepared as described previously (Shogren-Knaak et al. 2003).

than Gen5 alone (Tse et al. 1998b; Grant et al. 1999; Sendra et al. 2000). To assess the effects of chromatin context and Gcn5-associated proteins on the mechanism of acetylation of phosphorylated $\mathrm{H} 3$ amino-terminal tails, we performed a thorough in vitro kinetic analysis.

To test physiologically relevant HAT complexes, we compared the ability of recombinant yeast Gcn5 and the native SAGA complex to acetylate phosphorylated $\mathrm{H} 3$ amino-terminal tail peptides. In agreement with published results (Cheung et al. 2000; Lo et al. 2000), we found that serine 10 phosphorylation of $\mathrm{H} 3$ histone-tail peptides stimulated the rate of histone acetylation by Gcn5, although to a lesser degree than reported (Fig. 2A). However, similar analyses with peptides and the Gen5containing complex, SAGA, proved different than Gen5 alone. Phosphorylation of the histone H3 tail peptide on serine 10 actually decreased the rate of SAGA-mediated acetylation (Fig. 2B). This decrease is observed both at subsaturating and saturating substrate concentrations, with a 3.6-fold increase in $K_{m}$ for peptide, and a 1.3 -fold reduction in $k_{\text {cat }}$, contradicting the mild stimulation reported previously for SAGA at a single concentration and time point (Lo et al. 2000).

To test more physiologically relevant chromatin substrates, we generated histone H3 homogeneously phosphorylated at serine 10 using native chemical ligation (Shogren-Knaak et al. 2003; Shogren-Knaak and Peterson 2004) and incorporated it with other histones into nucleosomal arrays by salt dialysis (Carruthers et al. 1999). Like the peptide substrates, the rate of acetylation of the arrays by Gen 5 is stimulated by serine 10 phosphorylation (Fig. 3A). However, with the SAGA complex, serine 10 phosphorylation does not stimulate acetylation, and this lack of stimulation is observed over both saturating and subsaturating concentrations (Fig. 3B). Thus, our re- sults suggest that serine 10 phosphorylation of histone $\mathrm{H} 3$ does not stimulate acetylation by a native histone acetyltransferase complex on a chromatin substrate.

Recent structural studies of the catalytic domain of Tetrahymena Gcn5 in complex with a phosphorylated peptide and coenzyme A suggest that phosphopeptide binding occurs in a different manner than originally proposed and may be less stimulatory than originally reported (Clements et al. 2003). In the crystal structure of this ternary complex, the phosphate group adopts two separate orientations and does not interact with the arginine 164 residue, as originally proposed. Instead the phosphate group appears to facilitate a number of changes in the mode of peptide binding both distal and proximal to the phosphoserine residue, with threonine 11 adopting a number of new interactions. Altogether, there is a net increase in buried surface area for the phosphopeptide, and this would be expected to translate into increased binding affinity. Indeed, in kinetic analysis of this protein, there is roughly a threefold decrease in $K_{m}$ for the phosphopeptide, which denotes augmented phosphopeptide binding. Although in agreement with our own results with Gcn5 and peptides, this increase is less than the sixto tenfold originally reported. Interestingly, in their kinetic analysis of Tetrahymena Gcn5, the authors find that the maximal catalytic turnover rate, $k_{\mathrm{cat}}$, also decreases fourfold for the phosphopeptide. Since the enzymatic specificity constant is determined by $k_{\mathrm{cat}} / K_{m}$, the net result of these changes would not be expected to augment selectivity for phosphorylated peptides, which is contrary to results seen with yeast Gcn5.

Altogether, the available in vitro data shows that the acetyltransferase activity of recombinant Gcn5 may be stimulated by histone $\mathrm{H} 3$ serine 10 phosphorylation, while the native SAGA HAT complex is not stimulated 

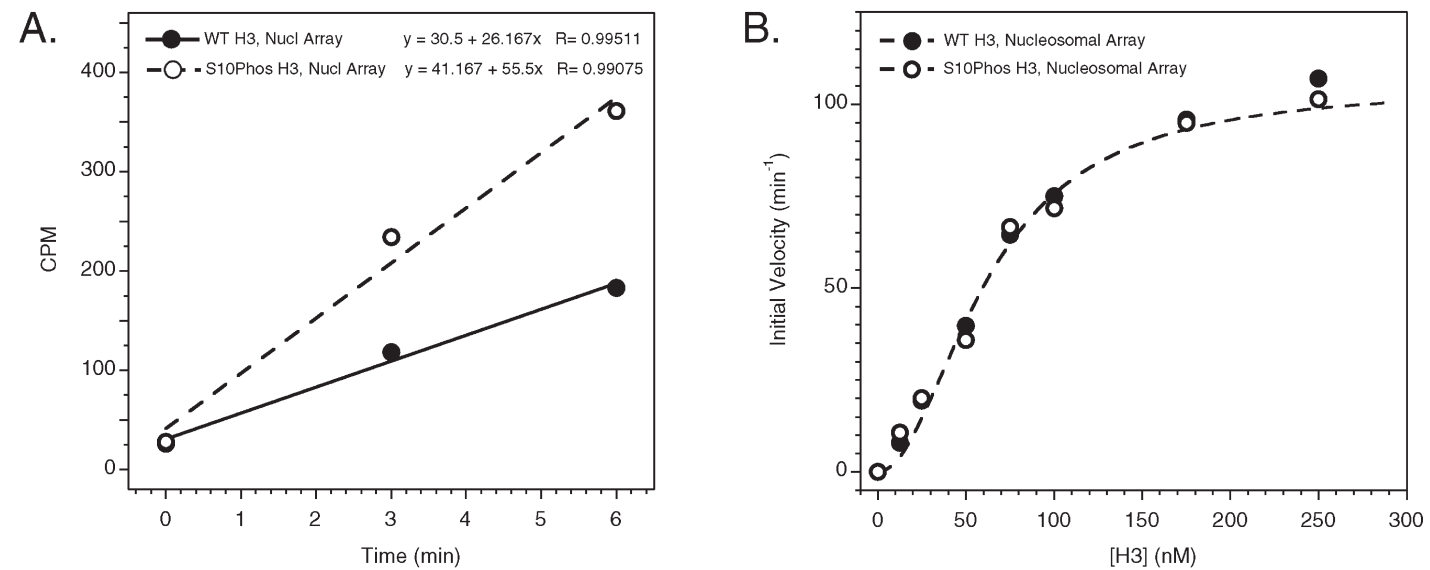

Figure 3. Histone H3 serine 10 phosphorylation stimulates the acetylation of nucleosomal arrays by Gen5p, but not by the yeast Gen5p-containing SAGA complex. (A) Comparison of Gen5p HAT activity on nucleosomal arrays containing unphosphorylated histone $\mathrm{H} 3$ (closed circles) or histone $\mathrm{H} 3$ phosphorylated on serine 10 (open circles). Histone acetyltransferase assays were performed with recombinant Gen5p $(2.45 \mu \mathrm{M})$ and nucleosomal arrays (100-nM histone H3), as described previously (Shogren-Knaak et al. 2003). The extent of histone acetylation was determined at multiple time points by counting the incorporation of tritiated acetate (counts per minute) using a scintillation counter. Each assay was repeated at least three times and the average counts per minute (CPM) is plotted as a function of time. (B) Comparison of SAGA HAT activity on phosphorylated (open circles) and unphosphorylated (closed circles) nucleosomal arrays. Histone acetyltransferase assays were performed with nucleosomal arrays (12.5-250-nM histone H3) and purified SAGA complex (4.9-9.8 nM). For each concentration of histone H3, the initial velocities were calculated by plotting the average incorporation of tritiated acetate as a function of time. Each assay was repeated at least three times and the average initial velocities are plotted as a function of histone $\mathrm{H} 3$ concentration. Nucleosomal arrays containing unphosphorylated histone $\mathrm{H} 3$ or histone $\mathrm{H} 3$ phosphorylated on S10 were prepared as described previously, using a native peptide ligation technique (ShogrenKnaak et al. 2003).

by histone phosphorylation of a peptide or a chromatin substrate. Why then does phosphorylation of serine 10 appear to enhance acetylation of $\mathrm{H} 3$ at some promoters in vivo? This coupling could be facilitated through a mechanism that does not rely on direct recruitment of Gen5 or activator proteins.

\section{MODULATING CHROMATIN STRUCTURE}

Histone phosphorylation at serine 10 could facilitate transcriptional activation by disrupting higher-order chromatin structure, thereby increasing the ability of transcription-related proteins to bind. Beyond the basic structure of the nucleosome, neighboring nucleosomes can interact to form a 30-nm chromatin fiber. These fibers can associate to generate 100-400-nm fibers, which then may adopt even higher orders of structure (Hayes and Hansen 2001). The charged and bulky nature of the phosphate group could lead to disruption of chromatin structure at any of these levels, either directly or indirectly. Because of the relatively high positive charge of the $\mathrm{H} 3$ histone tail $(+14)$, any effect of phosphorylation $(-2$ charges $)$ would probably result from the disruption or generation of specific binding contacts, instead of a bulk change in charge.

In vitro studies of nucleosomal arrays support the idea that changes to histone tails can influence chromatin structure. Nucleosomal arrays can be assembled from histones lacking amino-terminal tails, and these arrays prove defective in their ability both to fold into 30-nm fibers and to self-associate into 100-400-nm fibers (Fletcher and Hansen 1995; Schwarz et al. 1996). Furthermore, posttranslational modifications can disrupt higher-order structure. Nucleosomal arrays generated from hyperacetylated histones are also defective in folding into 30$\mathrm{nm}$ fibers and are stimulatory toward transcription in vitro (Tse et al. 1998a).

Evidence that histone phosphorylation might directly influence chromatin structure comes from studies of mitotic chromatin. During mitosis, when H3 is highly phosphorylated, the amount of DNA that can be cross-linked to histone $\mathrm{H} 3$ by $\mathrm{UV}$ irradiation is decreased relative to interphase levels. This loss is partially rescued by treating the mitotic cells with a kinase inhibitor, suggesting that kinase activity somehow leads to structural changes at the chromatin level. Moreover, the effect of inhibiting kinase activity on chromatin structure is apparent at a gross level, as disruption of mitotic condensation is visible microscopically (Sauve et al. 1999).

To more directly test the effects of histone H3 serine 10 phosphorylation on chromatin structure, we have assembled nucleosomal arrays containing homogeneously phosphorylated H3 histones and characterized these arrays biophysically using sedimentation analysis. In TE buffer, conditions where nucleosomal arrays are expected to adopt an extended "beads-on-a-string" conformation, arrays with or without H3 serine 10 phosphorylation display a similar, relatively tight distribution of sedimentation coefficients (Fig. 4A). These data reflect most directly on the similarity of the nucleosome structure and 

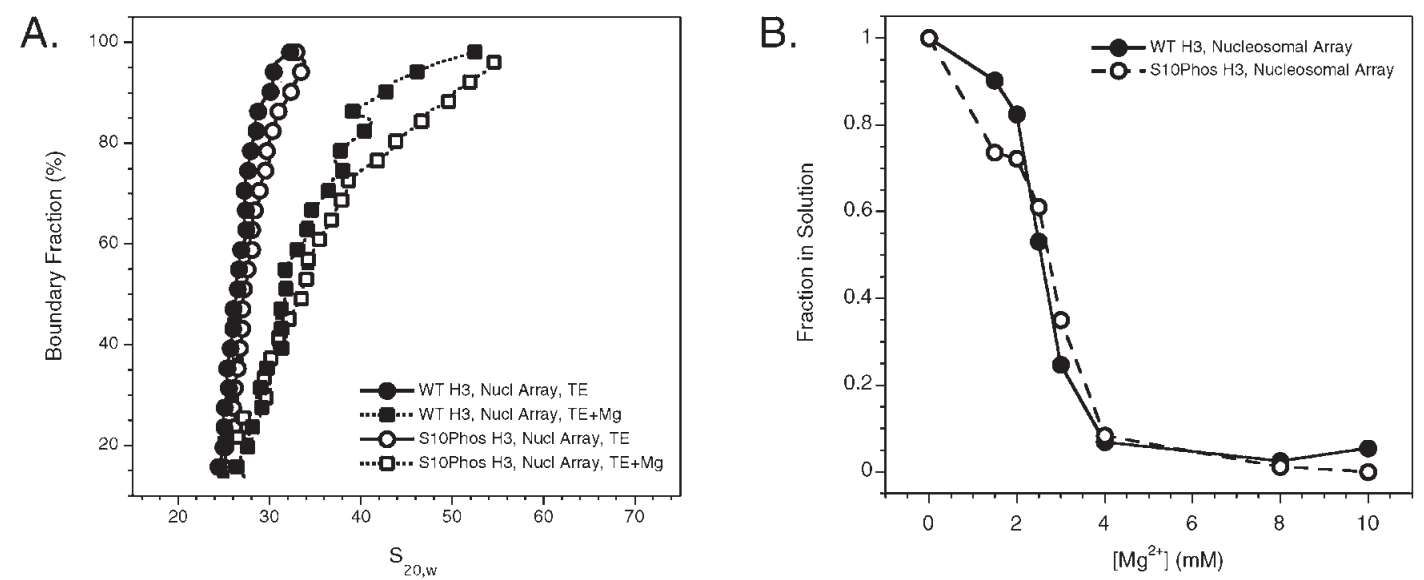

Figure 4. Histone H3-S10 phosphorylation does not effect higher-order chromatin structure. Experiments were performed with nucleosomal arrays assembled from 208-12 5S rDNA template, recombinant Xenopus H2A, H2B, and H4 histone protein, and either recombinant Xenopus H3 histone or ligated H3 histone-containing serine phosphorylated at position 10 (Shogren-Knaak et al. 2003). (A) Intramolecular folding of a nucleosomal array is not changed by histone $\mathrm{H} 3$ serine 10 phosphorylation. The sedimentation velocities of phosphorylated (open circles) and nonphosphorylated (closed circles) nucleosomal arrays were determined at $20^{\circ} \mathrm{C}$ in $\mathrm{TE}$ in the presence or absence of $1.75 \mathrm{mM} \mathrm{MgCl}_{2}$ (Horn et al. 2002). The $G(s)$ distributions were calculated by the method of van Holde and Weischet (van Holde and Weischet 1978) and corrected to water at $20^{\circ} \mathrm{C}$. $(B)$ Intermolecular association of a nucleosomal array is not changed by histone $\mathrm{H} 3$ serine 10 phosphorylation. Nucleosomal arrays were incubated in TE containing increasing amounts of $\mathrm{MgCl}_{2}$. After $15 \mathrm{~min}$ at room temperature, samples were centrifuged for $10 \mathrm{~min}$ at 14,000g, and the concentration of array in the supernatant was determined by measurement of absorption at $260 \mathrm{~nm}$. Shown is the fraction of array in solution as a function of $\mathrm{MgCl}_{2}$ concentration.

argue that serine 10 phosphorylation of histone $\mathrm{H} 3$ does not dramatically change the manner in which DNA is wrapped around the histone octamer nor how histone octamers become distributed onto the DNA. With the addition of 1.75-mM divalent magnesium cation, which promotes intramolecular nucleosome-nucleosome interactions, phosphorylated and unphosphorylated arrays show similar sedimentation properties (Fig. 4A). Both arrays adopt a distribution of $\mathrm{S}$ values from 29-55S, with $55 \mathrm{~S}$ reflecting fully folded, 30-nm fibers. Thus, H3 serine 10 phosphorylation does not appear to disrupt tailmediated chromatin folding. Finally, under concentrations of divalent magnesium above $2 \mathrm{~mm}$, conditions that promote reversible intermolecular nucleosomal array interactions, phosphorylated and nonphosphorylated arrays demonstrate similar sedimentation properties (Fig. 4B), suggesting that $\mathrm{H} 3$ serine 10 phosphorylation also does not disrupt tail-mediated internucleosomal array interactions.

Altogether, our sedimentation studies suggest that phosphorylation of serine 10 on histone $\mathrm{H} 3$ does not have a discernible effect on chromatin structure. Consistent with this conclusion is our previous work showing that SWI-SNF-mediated remodeling of nucleosomal arrays is not sensitive to histone phosphorylation (Shogren-Knaak et al. 2003), and recent results from the Richmond lab demonstrating that loss of histone H3 amino-terminal tails is not sufficient to disrupt chromatin folding (Dorigo et al. 2003). Nonetheless, histone H3 serine 10 phosphorylation may still exert a disruptive effect on chromatin structure in the context of additional histone modifications, including the high level of basal tail acetylation, or in combination with other tail phosphorylation marks (H3-S28, H3-T11, H3-T3, H4-S1, H2A-S1, H2B-S33) that occur during mitosis, meiosis, or transcriptional activation.

\section{DECREASING THE BINDING AFFINITY OF TRANSCRIPTIONAL REPRESSORS}

Several recent studies suggest an additional mechanism by which histone $\mathrm{H} 3$ phosphorylation may mediate transcriptional activation, by directly disrupting the binding of transcriptional repressor proteins. During mitosis, removal of the heterochromatin protein HP1 is preceded by histone $\mathrm{H} 3$ serine 10 phosphorylation, and phosphorylation is believed to directly promote loss of HP1 binding (Fischle et al. 2003a). However, the factors required to remove HP1 appear to be more complicated than just phosphorylation, as it seems that both serine 10 phosphorylation and lysine 14 acetylation are required for HP1 loss (Mateescu et al. 2004). The importance of acetylation hints at a potential connection to transcriptional activation. Indeed it has been found that HP1 plays a role in Rbmediated transcriptional repression of inducible genes (Nielsen et al. 2001), and phosphorylation in addition to acetylation may play a direct role in relieving this repression.

This strategy of derepressing genes by phosphorylation of the histone H3 tail may also apply to other transcriptional repressor proteins. Kouzarides and coworkers have shown that acetylation of histone $\mathrm{H} 3$ peptides at multiple sites, or phosphorylation of threonine 3 , serine 10 , or 
threonine 11, is sufficient to disrupt binding of the INHAT repressor complex (Schneider et al. 2004). The INHAT complex is a chromatin-associated complex that may inhibit acetylation of histones by physically masking acetylating sites (Seo et al. 2002). While loss of INHAT binding due to histone phosphorylation has not been confirmed in vivo, it does provide a mechanism in which phosphorylation and acetylation are coupled, and thus provides an appealing model for how histone phosphorylation might facilitate transcription.

\section{CONCLUSION}

Histone $\mathrm{H} 3$ phosphorylation at serine 10 is an important means of facilitating transcription of a number of inducible genes. However, the mechanism for this coupling remains controversial. Initial in vitro studies with Gen5 and peptides suggested that transcriptional stimulation might be accomplished by augmented binding of Gen5 to H3-S10 phosphorylated histone tails. However, studies with more physiologically relevant Gcn5-containing complexes and nucleosomal arrays do not show an increase in binding affinity toward H3-S10-phosphorylated histone, and alternative mechanisms are now being uncovered. Structural studies with H3-S10-phosphorylated nucleosomal arrays demonstrate that histone $\mathrm{H} 3$ phosphorylation on serine 10 is not sufficient to disrupt higher-order chromatin structure. Alternatively, preliminary studies suggest that H3-S10 phosphorylation may disrupt binding of transcriptional repressors and provides an attractive mechanism by which H3-S10 phosphorylation might facilitate histone acetylation and transcription. The complex interplay of phosphorylation, acetylation, and methylation of histone tails remains a rich area of inquiry and continued studies will provide a deeper understanding of transcription and other crucial biological processes.

\section{ACKNOWLEDGMENTS}

Our studies on histone phosphorylation were supported by a grant from the NCI to C.L.P. (CA82834), and postdoctoral fellowships from the NIH to M.S-K. (F32 AI10611) and from the Leukemia and Lymphoma Society of America to C.J.F.

\section{REFERENCES}

Allegra P., Sterner R., Clayton D.F., and Allfrey V.G. 1987. Affinity chromatographic purification of nucleosomes containing transcriptionally active DNA sequences. J. Mol. Biol. 196: 379 .

Becker P.B. and Horz W. 2002. ATP-dependent nucleosome remodeling. Annu. Rev. Biochem. 71: 247.

Carruthers L.M., Tse C., Walker K.P., III, and Hansen J.C. 1999. Assembly of defined nucleosomal and chromatin arrays from pure components. Methods Enzymol. 304: 19.

Chen T.A. and Allfrey V.G. 1987. Rapid and reversible changes in nucleosome structure accompany the activation, repression, and superinduction of murine fibroblast protooncogenes c-fos and c-myc. Proc. Natl. Acad. Sci. 84: 5252.

Cheung P., Tanner K.G., Cheung W.L., Sassone-Corsi P., Denu J.M., and Allis C.D. 2000. Synergistic coupling of histone H3 phosphorylation and acetylation in response to epidermal growth factor stimulation. Mol. Cell 5: 905.
Clements A., Poux A.N., Lo W.S., Pillus L., Berger S.L., and Marmorstein R. 2003. Structural basis for histone and phosphohistone binding by the GCN5 histone acetyltransferase. Mol. Cell 12: 461.

Dhalluin C., Carlson J.E., Zeng L., He C., Aggarwal A.K., and Zhou M.M. 1999. Structure and ligand of a histone acetyltransferase bromodomain. Nature 399: 491.

Dorigo B., Schalch T., Bystricky K., and Richmond T.J. 2003. Chromatin fiber folding: Requirement for the histone $\mathrm{H} 4 \mathrm{~N}$ terminal tail. J. Mol. Biol. 327: 85.

Fischle W., Wang Y., and Allis C.D. 2003a. Binary switches and modification cassettes in histone biology and beyond. Nature 425: 475 .

2003b. Histone and chromatin cross-talk. Curr. Opin. Cell Biol. 15: 172.

Fletcher T.M. and Hansen J.C. 1995. Core histone tail domains mediate oligonucleosome folding and nucleosomal DNA organization through distinct molecular mechanisms. J. Biol. Chem. 270: 25359.

Grant P.A., Eberharter A., John S., Cook R.G., Turner B.M., and Workman J.L. 1999. Expanded lysine acetylation specificity of Gen5 in native complexes. J. Biol. Chem. 274: 5895.

Hayes J.J. and Hansen J.C. 2001. Nucleosomes and the chromatin fiber. Curr. Opin. Genet. Dev. 11: 124.

Horn P.J., Crowley K., Carruthers L.M., Hansen J.C., and Peterson C.L. 2002. The SIN domain of the histone octamer is essential for intramolecular folding of nucleosomal arrays. Nat. Struct. Biol. 9: 167.

Lo W.S., Duggan L., Emre N.C., Belotserkovskya R., Lane W.S., Shiekhattar R., and Berger S.L. 2001. Snf1-A histone kinase that works in concert with the histone acetyltransferase Gcn5 to regulate transcription. Science 293: 1142.

Lo W.S., Trievel R.C., Rojas J.R., Duggan L., Hsu J.Y., Allis C.D., Marmorstein R., and Berger S.L. 2000. Phosphorylation of serine 10 in histone $\mathrm{H} 3$ is functionally linked in vitro and in vivo to Gen5-mediated acetylation at lysine 14. Mol. Cell 5: 917.

Luger K., Mader A.W., Richmond R.K., Sargent D.F., and Richmond T.J. 1997. Crystal Structure of the nucleosome core particle at $2.8 \AA$ \& resolution. Nature 389: 251.

Mahadevan L.C., Willis A.C., and Barratt M.J. 1991. Rapid histone $\mathrm{H} 3$ phosphorylation in response to growth factors, phorbol esters, okadaic acid, and protein synthesis inhibitors. Cell 65: 775 .

Mateescu B., England P., Halgand F., Yaniv M., and Muchardt C. 2004 . Tethering of HP1 proteins to chromatin is relieved by phosphoacetylation of histone H3. EMBO Rep. 5: 490.

Ng H.H., Feng Q., Wang H., Erdjument-Bromage H., Tempst P., Zhang Y., and Struhl K. 2002. Lysine methylation within the globular domain of histone H3 by Dot1 is important for telomeric silencing and Sir protein association. Genes Dev. 16: 1518

Nielsen P.R., Nietlispach D., Mott H.R., Callaghan J., Bannister A., Kouzarides T., Murzin A.G., Murzina N.V., and Laue E.D. 2002. Structure of the HP1 chromodomain bound to histone $\mathrm{H} 3$ methylated at lysine 9. Nature 416: 103.

Nielsen S.J., Schneider R., Bauer U.M., Bannister A.J., Morrison A., O'Carroll D., Firestein R., Cleary M., Jenuwein T., Herrera R.E., and Kouzarides T. 2001. Rb targets histone H3 methylation and HP1 to promoters. Nature 412: 561.

Nowak S.J. and Corces V.G. 2000. Phosphorylation of histone H3 correlates with transcriptionally active loci. Genes Dev. 14: 3003 .

Prigent C. and Dimitrov S. 2003. Phosphorylation of serine 10 in histone H3, what for? J. Cell Sci. 116: 3677.

Rojas J.R., Trievel R.C., Zhou J., Mo Y., Li X., Berger S.L., Allis C.D., and Marmorstein R. 1999. Structure of Tetrahymena GCN5 bound to coenzyme A and a histone $\mathrm{H} 3$ peptide. $\mathrm{Na}$ ture 401: 93.

Sassone-Corsi P., Mizzen C.A., Cheung P., Crosio C., Monaco L., Jacquot S., Hanauer A., and Allis C.D. 1999. Requirement of Rsk-2 for epidermal growth factor-activated phosphorylation of histone H3. Science 285: 886.

Sauve D.M., Anderson H.J., Ray J.M., James W.M., and 
Roberge M. 1999. Phosphorylation-induced rearrangement of the histone $\mathrm{H} 3 \mathrm{NH} 2$-terminal domain during mitotic chromosome condensation. J. Cell Biol. 145: 225.

Schneider R., Bannister A.J., Weise C., and Kouzarides T. 2004. Direct binding of INHAT to $\mathrm{H} 3$ tails disrupted by modifications. J. Biol. Chem. 279: 23859.

Schwarz P.M., Felthauser A., Fletcher T.M., and Hansen J.C. 1996. Reversible oligonucleosome self-association: Dependence on divalent cations and core histone tail domains. Biochemistry 35: 4009.

Sendra R., Tse C., and Hansen J.C. 2000. The yeast histone acetyltransferase A2 complex, but not free Gcn5p, binds stably to nucleosomal arrays. J. Biol. Chem. 275: 24928.

Seo S.B., Macfarlan T., McNamara P., Hong R., Mukai Y., Heo S., and Chakravarti D. 2002. Regulation of histone acetylation and transcription by nuclear protein $\mathrm{pp} 32$, a subunit of the INHAT complex. J. Biol. Chem. 277: 14005.

Shogren-Knaak M.A. and Peterson C.L. 2004. Creating designer histones by native chemical ligation. Methods Enzymol. 375: 62.

Shogren-Knaak M.A., Fry C.J., and Peterson C.L. 2003. A native peptide ligation strategy for deciphering nucleosomal histone modifications. J. Biol. Chem. 278: 15744.

Soloaga A., Thomson S., Wiggin G.R., Rampersaud N., Dyson M.H., Hazzalin C.A., Mahadevan L.C., and Arthur J.S. 2003. MSK2 and MSK1 mediate the mitogen- and stress-induced phosphorylation of histone $\mathrm{H} 3$ and HMG-14. EMBO J. 22: 2788.

Thomson S., Clayton A.L., and Mahadevan L.C. 2001. Indepen- dent dynamic regulation of histone phosphorylation and acetylation during immediate-early gene induction. Mol. Cell 8: 1231 .

Tse C., Sera T., Wolffe A.P., and Hansen J.C. 1998a. Disruption of higher-order folding by core histone acetylation dramatically enhances transcription of nucleosomal arrays by RNA polymerase III. Mol. Cell. Biol. 18: 4629.

Tse C., Georgieva E.I., Ruiz-Garcia A.B., Sendra R., and Hansen J.C. 1998b. Gcn5p, a transcription-related histone acetyltransferase, acetylates nucleosomes and folded nucleosomal arrays in the absence of other protein subunits. J. Biol. Chem. 273: 32388 .

van Holde K.E. and Weischet W.O. 1978. Boundary analysis of sedimentation-velocity experiments with monodisperse and paucidisperse solutes. Biopolymers 17: 1387.

van Leeuwen F., Gafken P.R., and Gottschling D.E. 2002. Dot1p modulates silencing in yeast by methylation of the nucleosome core. Cell 109: 745.

Wiggin G.R., Soloaga A., Foster J.M., Murray-Tait V., Cohen P., and Arthur J.S. 2002. MSK1 and MSK2 are required for the mitogen- and stress-induced phosphorylation of CREB and ATF1 in fibroblasts. Mol. Cell. Biol. 22: 2871.

Woodcock C.L. and Dimitrov S. 2001. Higher-order structure of chromatin and chromosomes. Curr. Opin. Genet. Dev. 11: 130.

Yaffe M.B. and Elia A.E. 2001. Phosphoserine/threonine-binding domains. Curr. Opin. Cell Biol. 13: 131.

Zhang L., Eugeni E.E., Parthun M.R., and Freitas M.A. 2003. Identification of novel histone post-translational modifications by peptide mass fingerprinting. Chromosoma 112: 77 . 


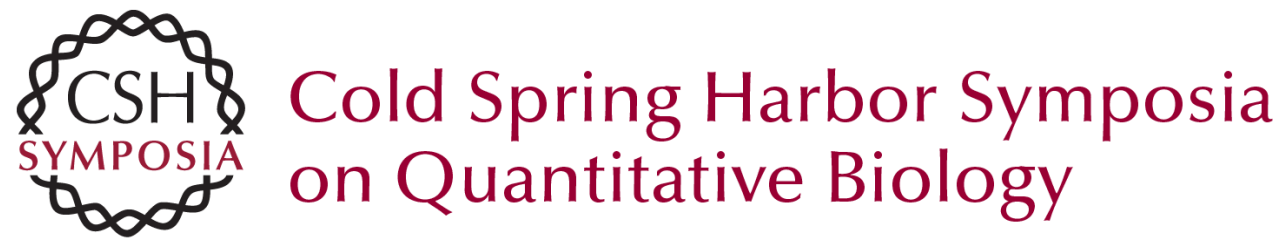

\title{
Histone H3 Amino-Terminal Tail Phosphorylation and Acetylation: Synergistic or Independent Transcriptional Regulatory Marks?
}

\author{
C.J. FRY, M.A. SHOGREN-KNAAK and C.L. PETERSON
}

Cold Spring Harb Symp Quant Biol 2004 69: 219-226

Access the most recent version at doi:10.1101/sqb.2004.69.219

$\begin{array}{ll}\text { References } & \text { This article cites } 43 \text { articles, } 17 \text { of which can be accessed free at: } \\ \text { http://symposium.cshlp.org/content/69/219.full.html\#ref-list-1 }\end{array}$

\section{License}

Email Alerting Receive free email alerts when new articles cite this article - sign up in Service the box at the top right corner of the article or click here. 